\title{
Visual systems \& control on polynomial space and its application to sloshing problems
}

\author{
Satoru Sakai and Masakazu Sato
}

\begin{abstract}
This paper proposes a novel approach for camera based modeling and control for a large class of continuous systems and the validity is confirmed by liquid sloshing experiments. It is an unsolved problem to design a model based control in non-planar sloshing cases. This is because the whole shape of the liquid surface is a complex curve (a set of an infinite number of points) in coordinate spaces. This paper solves this problem. First, the whole shape of the liquid surface corresponding to the output measured by a camera is a single point in a polynomial space as well as the input and the state. Second, without any physical parameter identification, input-output modeling on polynomial space, unlike existing types of modeling, captures the whole dynamics even in non-planar sloshing cases and is linked to design of implementable controllers. Finally, in the presence of occlusion, the non-planar sloshing are controlled well by state estimation on polynomial space without adding any image processing technology.
\end{abstract}

Index Terms-Visual systems \& control, Hilbert space, continuous systems

\section{INTRODUCTION}

Visual feedback will be a key technology in this century. Especially, the intersection of two technologies, control systems technology and image processing technology, has become a focus. This paper proposes a novel approach for camera based modeling and control. The proposed approach is directly applicable to a large class of continuous systems (continuum system) that is too difficult for existing approaches. The validity of the proposed approach is confirmed by liquid sloshing experiments which are important in the fields of civil, chemical and aerospace engineering as well as robotics and automation [1] [2] [3].

Fundamental problems in modeling and control of liquid sloshings are unsolved [4]. Physical modeling [5] [6] has been discussed to capture the whole dynamics including the interconnection between the fluid dynamics (liquid dynamics) [7] and the mechanical dynamics (the tank dynamics, the vibration absorber dynamics and the other structural dynamics) [8], assuming simple boundary conditions. Physical modeling also captures the input (e.g., the driving force for the tank dynamics) in coordinate spaces ( $n$-spaces) $\mathbb{R}^{n}$ in many cases or $\mathbb{C}^{n}$ in case of the Euler formula expression $A \exp (j \omega)$. However, the resultant input-state signal mapping is nonlinear ${ }^{1}$ and physical modeling is too complex for applying existing designs in nonlinear systems \& control (e.g., [9] [10]) even in simple boundary conditions.

S. Sakai and M. Sato are with the Department of Mechanical Engineering, Shinshu University, Wakasato, Nagano, Japan. E-mail: satorusakai@gakushikai.jp

${ }^{1}$ The corresponding state space equations are not discussed here because the equations can be nonlinear even if the input-state signal mapping is linear [9].
Without assuming simple boundary conditions, numerical modeling [11] [12] can capture the whole dynamics, namely, the computational fluid dynamics interconnected to the mechanical dynamics forced by the input. But it is clear that numerical modeling cannot be linked to design of implementable controllers due to the heavy computational cost.

Equivalent mechanical modeling [2] [5] [13] [14] [15], is a practical reduction from the original liquid dynamics to a mechanical dynamics in which forces and moments acting on the tank wall are equivalent to those in the original liquid dynamics. Equivalent mechanical modeling is less complex and may be linked to design of implementable controllers. For example, in planar sloshing cases [5], the original liquid dynamics is reduced to single-pendulum dynamics [16] [17] [18] or mass-damper-spring dynamics [19] [20]. Since the interconnection between these reduced mechanical dynamics and the tank dynamics generates another mechanical dynamics, equivalent mechanical modeling is not complex for applying existing designs in mechanical systems \& control (e.g., [21] [22]). In addition, the designed controllers are implementable because the mechanical configuration, namely, the liquid inclination has been already measured by a level sensor in planar sloshing cases.

However, in non-planar sloshing cases [5] [23], the designed controllers are not implementable. Although the original liquid dynamics is reduced to spherical pendulum dynamics or multiple-pendulum dynamics, these mechanical configurations, that is, the whole shape of the liquid surface, is not always measured by a few level sensors.

In the absence of occlusion (visual obstacles), the whole shape of the liquid surface is measured by a camera instead of a few level sensors. However, to our knowledge, for control, the vertical displacements of only a few points (e.g., an edge point contacting to a tank wall) on the liquid surface are measured by image processing technology [19]. That is, a camera just plays the role of a few level sensors and the whole shape of the liquid surface is never used. This is because the whole shape of the liquid surface is a set of an infinite number of points in the coordinate space $\mathbb{R}^{n}$. If one increases the number of measured points on the liquid surface, equivalent mechanical modeling is not linked to design of implementable controllers due to the heavy computational cost again.

On the other hand, conventional input-output modeling (system identification) on coordinate space [2] [24] [25] $\mathbb{R}^{n}$ in case of a state space expression or $\mathbb{C}^{n}$ in case of a transfer function expression is almost always linked to design of implementable controllers for linear time invariant (LTI) 
TABLE I

A CLASSIFICATION OF MODELING, CONTROL DESIGN AND IMPLEMENTATION FOR LIQUID SLOSHINGS

\begin{tabular}{l|l||l}
\hline Modeling & \multicolumn{1}{||}{ Control design } \\
\hline Physical modeling & Whole dynamics , white-box on $\mathbb{R}^{n}, \mathbb{C}^{n}$ & Implementation \\
Numerical modeling & Whole dynamics & - \\
Equivalent mechanical modeling & Whole dynamics , white-box on $\mathbb{R}^{n}$ & Mechanical systems \& control \\
Conventional input-output modeling & Partial dynamics , black-box on $\mathbb{R}^{n}, \mathbb{C}^{n}$ & Imput-output/linear systems \& control \\
Proposed visual modeling & Whole dynamics , black-box on $P_{n-1}[-1,1]$ & Input-output/linear systems \& control \\
\hline
\end{tabular}

$+=$ "Numerical modeling captures chaotic sloshing (non-planar sloshing with overturned waves) cases that the other types of modeling do not."

* = "Equivalent mechanical modeling is linked to design of implementable controllers in planar sloshing cases. "

$\diamond=$ "Conventional input-output modeling captures planar sloshing cases as long as the liquid inclination and its velocity are small."

systems. However, conventional input-output modeling cannot capture the whole dynamics because a signal mapping from the input to any liquid surface displacement corresponding to the output is "strongly nonlinear" on coordinate space in nonplanar sloshing cases. Indeed, non-planar sloshing is called nonlinear sloshing even in recent works [5] [7].

Overall, it is an open problem to develop new type of modeling which captures the whole dynamics of both the liquid and the tank even in non-planar sloshing cases and which is linked to design of implementable controllers. Similar problems exist in other continuous systems.

This paper proposes a novel approach to solve this problem and visual systems \& control on polynomial space is introduced. Since a polynomial space is isomorphic to a coordinate space $\mathbb{R}^{n}$ [26], it is possible to execute input-output modeling (system identification) on polynomial space just as on coordinate space. This modeling is justified if a signal mapping from the input to the whole shape of the liquid surface is "almost linear" on polynomial space even when a signal mapping from the same input to any liquid surface displacement is "strongly nonlinear" on coordinate space.

It is NOT a main contribution of this paper to see the whole shape of the liquid surface as a polynomial (a single point in the polynomial space). This is because the whole shapes of some continuous systems [27] [28] are already seen as the specific functions (not polynomials but the Bessel function [5] and the Riesz function [2] etc.) from the viewpoint of dynamical systems without inputs. In a similar way, the whole shapes of other continuous systems [29] are seen as the classical Fourier series from the viewpoint of signals instead of dynamical systems.

In this paper, from the viewpoint of dynamical systems with inputs (signal mapping), not only the whole shape of the liquid surface corresponding to the output but also the input and the state are polynomials. The output is a visible polynomial measured by a camera and the input is known and a visualizable polynomial. The state is unknown and never visualizable but exists and causes the liquid sloshings.

Without any physical parameter identification, the whole dynamics of both the liquid and the tank is captured by executing input-output modeling (system identification) on polynomial space even if the output is a high-degree polynomial in non-planar sloshing cases caused by complex boundary conditions or the inputs. The proposed visual modeling (the input-output modeling on polynomial space) is easily linked to design of implementable controllers since it is also possible to execute existing control designs on polynomial space just as on coordinate space again. In this paper, the validity of the proposed visual modeling is confirmed by liquid sloshing experiments in the absence of occlusion at first.

In general, occlusion is an important issue in visual feedbacks. The closed-loop stability or control performance is lost in the presence of occlusion between a camera and a plant. Since the shape and size of the visual obstacles are unknown in actual control situations, their effects are difficult to be rejected by image processing technology. To solve this occlusion problem, in this paper, the camera occlusion effects are modeled as disturbances in the polynomial space and then rejected by executing state estimation on polynomial space. In other words, the occlusion problem is solved by control systems technology instead of image processing technology. This is a new result and important at the intersection of the two technologies.

The rest of this paper is organized as follows. In Section II, time-varying polynomial space is reviewed. In Section III, the modeling of the liquid sloshing is numerically and experimentally discussed on polynomial space. It is confirmed that the proposed visual modeling captures the whole dynamics even in non-planar sloshing cases. In Section VI, the control of the liquid sloshing is numerically and experimentally discussed on polynomial space. It is confirmed that the proposed visual modeling is linked to design of implementable controllers. Furthermore, the camera occlusion effects are rejected by executing state estimation on polynomial space. Finally, this paper is concluded in Section V.

\section{TIME-VARYING POLYNOMIAL SPACE}

This section reviews a polynomial space in the time-varying version. Though a polynomial space is isomorphic to a coordinate space, from the viewpoint of visual systems \& control, it is not relevant to identify both spaces.

Let us consider a finite dimensional space of polynomials of degree at most $n-1(n \in \mathbb{N})$ on the real interval $[a, b]$ which is denoted by $P_{n-1}[a, b]$. Also, let us suppose that a time-varying polynomial $f(r, k) \in P_{n-1}[a, b]$ depends on the discrete time instant $k \in \mathbb{Z}_{+}:=\{0\} \cup \mathbb{N}$ as well as the horizontal space parameter $r \in[a, b]$.

The polynomial space $P_{n-1}[a, b]$ is a Hilbert space and is closed with respect to the standard addition and scalar 


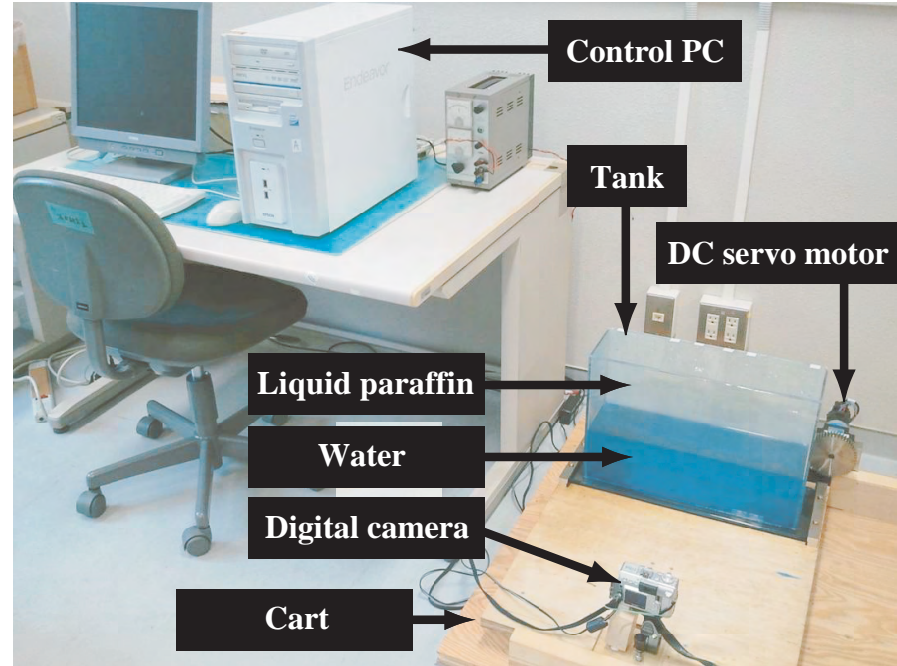

Fig. 1. Experimental setup.

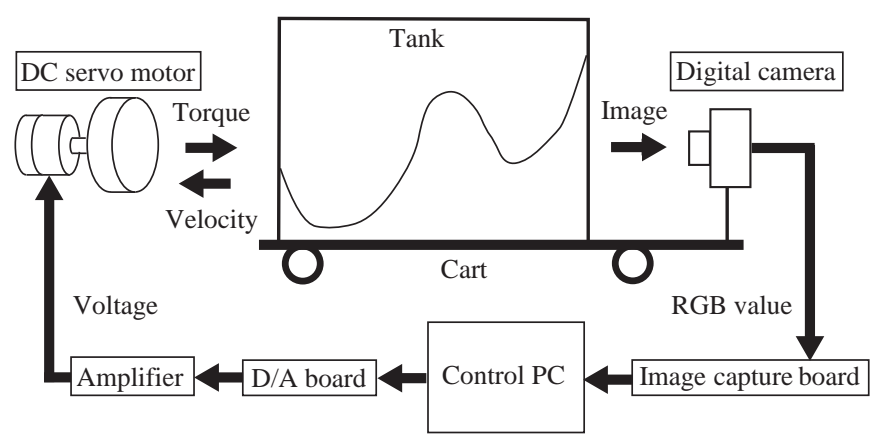

Fig. 2. System configuration.

multiplication. Furthermore, the following inner product

$$
\langle f(r, k), g(r, k)\rangle:=\int_{a}^{b} f(r, k) g(r, k) d r \in \mathbb{R}
$$

is well-defined for $\forall f(r, k), \forall g(r, k) \in P_{n-1}[a, b]$ and brings the completeness with respect to the inner product norm $\|f(r, k)\|:=\sqrt{\langle f(r, k), f(r, k)\rangle}$.

The Legendre polynomials in a special polynomial space $P_{n-1}[-1,1]$

$$
L_{h}(r):=\frac{1}{2^{h} \cdot h !} \cdot \frac{d^{h}}{d r^{h}}\left(r^{2}-1\right)^{h}, h=0, \cdots, n-1
$$

generate a normalized orthogonal basis [30] in the order

$$
e_{m}(r):=\sqrt{\frac{2 m-1}{2}} L_{m-1}(r), \quad m=1, \cdots, n
$$

satisfying the property

$\left\langle e_{m_{i}}(r), e_{m_{j}}(r)\right\rangle=\left\{\begin{array}{ll}0 & \left(m_{i} \neq m_{j}\right) \\ 1 & \left(m_{i}=m_{j}\right)\end{array}, \quad m_{i}, m_{j}=1, \cdots, n\right.$.

Thus, a time-varying polynomial

$$
f(r, k)=\sum_{m=1}^{n}\left\langle f(r, k), e_{m}(r)\right\rangle e_{m}(r) \in P_{n-1}[-1,1]
$$

is expressed as $\left[f_{1}(k), f_{2}(k), \cdots, f_{n}(k)\right]^{\mathrm{T}}$ whose component has the form of

$$
f_{m}(k):=\left\langle f(r, k), e_{m}(r)\right\rangle .
$$

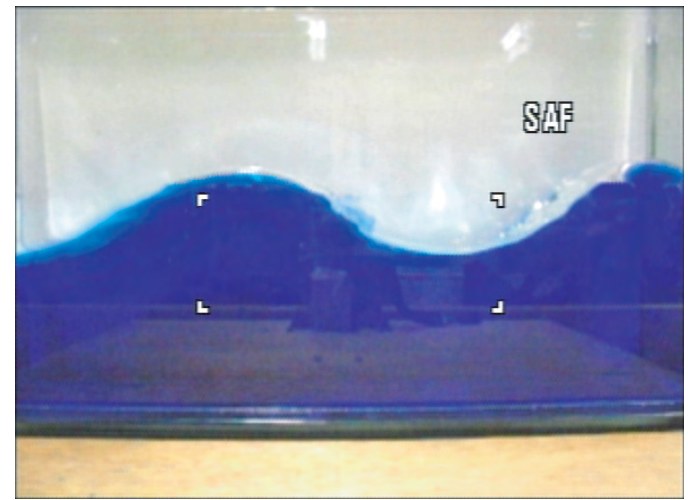

Fig. 3. Example image.

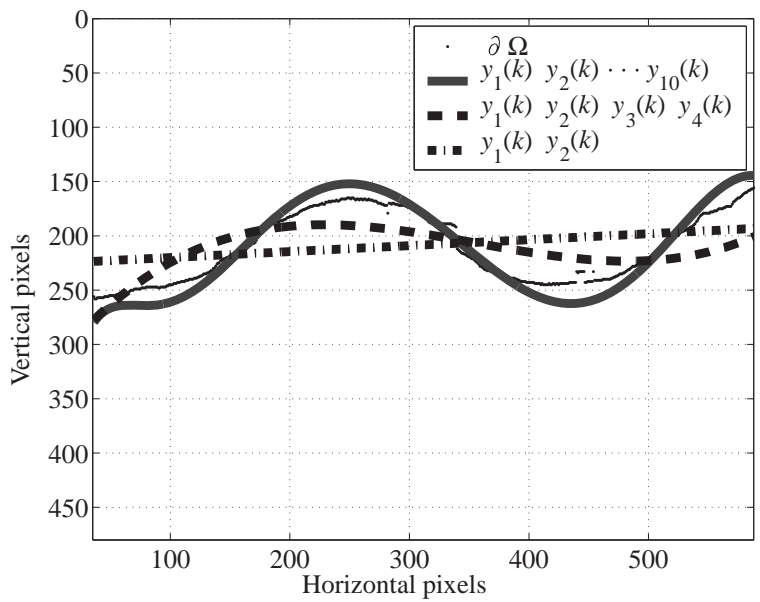

Fig. 4. Extracted features and outputs $y$ for the example image.

\section{INPUT-OUTPUT MODELING ON POLYNOMIAL SPACE}

In this section, a signal mapping between time-varying polynomial spaces is introduced and input-output modeling (system identification) to capture the whole dynamics is discussed numerically and experimentally.

\section{A. Experimental method (Modeling)}

A liquid surface at the discrete time instant $k$ is measured via the following feature extraction as an image processing technology and is modeled as an object in a polynomial space.

Let us define a Cartesian coordinate for every image in a digital camera and make each pixel center correspond to each lattice point $(r, s) \in \mathbb{Z} \times \mathbb{Z}$. Scanning along the horizontal $r$-axis and the vertical $s$-axis, the liquid surface is measured as

$$
\begin{aligned}
& \partial \Omega(r, k):= \max _{(r, s) \in \Omega(k)} s \\
& \Omega(k):=\quad\left\{(r, s) \mid r_{1} \leq R(r, s, k) \leq r_{2},\right. \\
& g_{1} \leq G(r, s, k) \leq g_{2}, \\
&\left.b_{1} \leq B(r, s, k) \leq b_{2}\right\}
\end{aligned}
$$

where $R(r, s, k), G(r, s, k), B(r, s, k)$ are the RGB-values of each pixel $(r, s)$ at the discrete time instant $k$ and $r_{j}, g_{j}, b_{j}(j=$ $1,2)$ are the corresponding thresholds as the features of the liquid whose boundary is the tank wall and the liquid surface $\partial \Omega(r, k)$. 

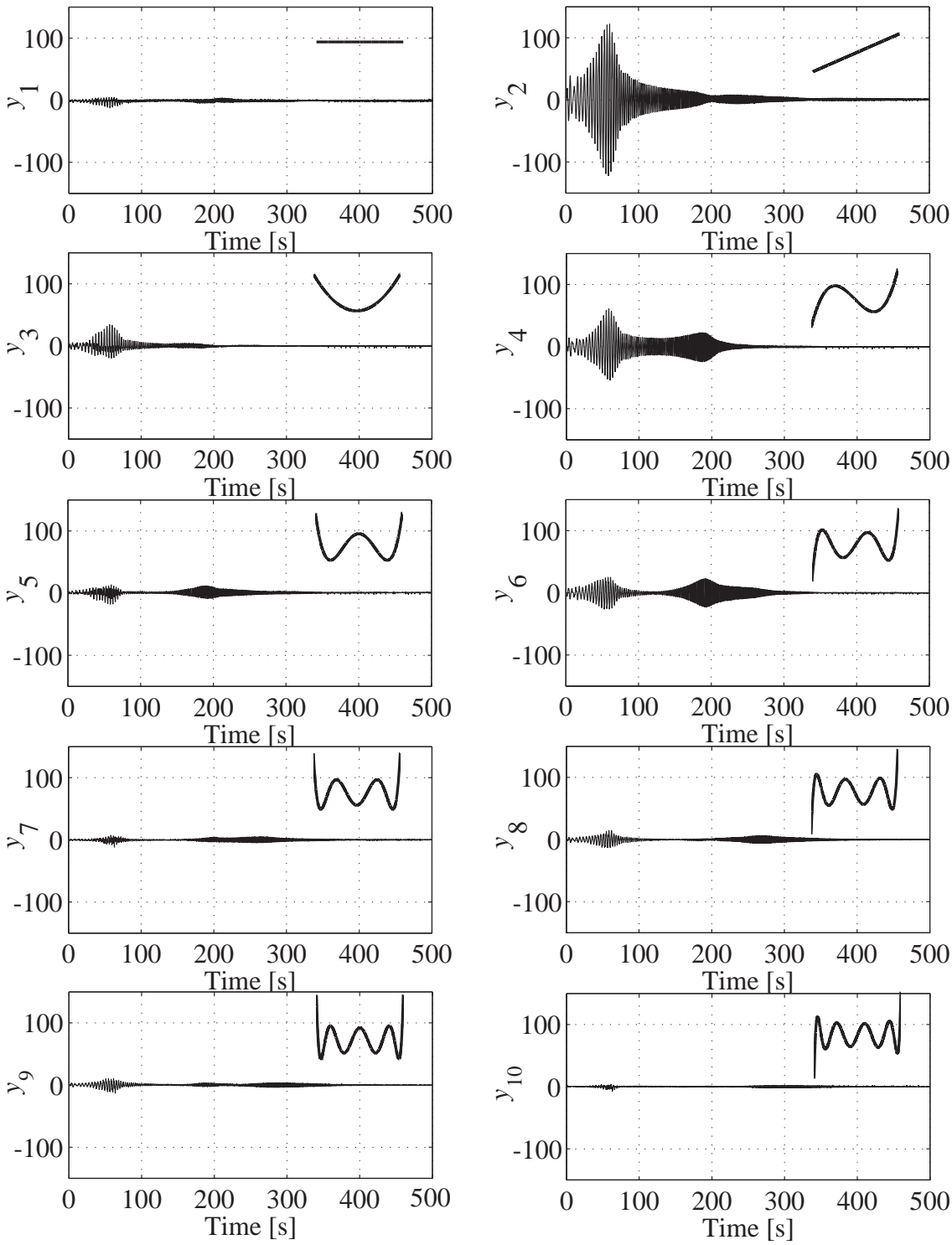

Fig. 5. Time-response of the output components $y_{1}, \cdots, y_{10}$.

By the projection theorem [26], the liquid surface $\partial \Omega(r, k)$ in a Hilbert space is projected to an $n_{y}-1$ th-degree polynomial as the output in the polynomial space. More precisely, based on the basis (1), the output is expressed as $\left[y_{1}(k), y_{2}(k), \cdots, y_{n_{y}}(k)\right]^{\mathrm{T}}$ whose component has the form of

$$
y_{m}(k)=\left\langle\partial \Omega(r, k), e_{m}(r)\right\rangle, m=1, \cdots, n_{y}
$$

via the scaling of the $r$-axis to match the interval $[-1,1]$ and the transition of the $s$-axis to make the horizontal surface be expressed as $[0, \cdots, 0]^{\mathrm{T}}$.

Consider a finite dimensional discrete time LTI system as a signal mapping between polynomial spaces and describe by

$$
\left\{\begin{aligned}
x(r, k+1) & =\mathcal{A} \circ x(r, k)+\mathcal{B} \circ u(r, k)+v(r, k), \\
y(r, k) & =\mathcal{C} \circ x(r, k)+\mathcal{D} \circ u(r, k)+w(r, k) \\
& \approx \partial \Omega(r, k)
\end{aligned}\right.
$$

where the state $x(r, k) \in P_{n_{x}-1}[-1,1]$ and the disturbance $v(r, k) \in P_{n_{x}-1}[-1,1]$ are the $n_{x}-1$ th-degree polynomials,

the input $u(r, k) \in P_{0}[-1,1]$ is the constant polynomial, the output $y(r, k) \in P_{n_{y}-1}[-1,1]$ and the disturbance $w(r, k) \in$ $P_{n_{y}-1}[-1,1]$ are the $n_{y}-1$ th-degree polynomials. The symbol $\circ$ denotes operation of the linear mappings $\mathcal{A}, \mathcal{B}, \mathcal{C}$ and $\mathcal{D}$ between the polynomial spaces.

Remark So many existing modeling and control methods (e.g., ARX, N4SID, LQG, $H_{\infty}$ and so on) are constructed on coordinate space $\mathbb{R}^{n}$. On the other hand, in abstract linear algebra, it is well known that a polynomial space $P_{n-1}[-1,1]$ is isomorphic to a coordinate space $\mathbb{R}^{n}$. That is, roughly speaking, the mathematical structure of the polynomial space $P_{n-1}[-1,1]$ and that of the coordinate space $\mathbb{R}^{n}$ are the same. This fact allows us to apply the same modeling and control methods to both spaces.

Remark Generally, since a mapping can not be defined before the domain and the co-domain are defined, the mapping depends on the choice of the domain and the co-domain. In other words, a system depends on the choice of the input 
space and the output space. In this sense, the proposed LTI system (3) choosing the polynomial spaces and any conventional LTI system choosing the coordinates spaces are always different objects even if the mappings $\mathcal{A}, \mathcal{B}, \mathcal{C}, \mathcal{D}$ in the state space expression of both LTI systems have the same matrix representations.

Remark In the proposed visual modeling, both the input $u$ and the state $x$ are polynomials as well as the output $y$. The output $y$ is a visible polynomial as the liquid surface but the input $u$ and the state $x$ are invisible polynomials as are the disturbances $v$ and $w$. Of course, the input $u$ is not unknown and visualizable but the state $x$ is never visualizable even in the absence of the disturbances $v$ and $w$. In this sense, the existence of the "state polynomial" is a key of this paper. Interestingly, we can imagine and estimate that the invisible polynomial $x$ behaves because polynomial spaces, unlike coordinate spaces, make it possible to draw the highdimensional (more than four-dimensional) state directly.

To identify the matrix representation of each mapping $\mathcal{A}, \mathcal{B}, \mathcal{C}, \mathcal{D}$, input-output modeling (system identification) is executed on polynomial space. Single-input and single-output (SISO) models $G_{1}(s), \cdots, G_{n_{y}}(s)$ from the input component to each output component is identified based on the auto regressive exogenous (ARX) method with the following chirp input

$u(r, k)=A_{u} \cos \left(\left(\omega_{1}+\frac{\omega_{2}-\omega_{1}}{M} T_{\mathrm{sam}} k\right) T_{\mathrm{sam}} k+\theta_{0}\right) e_{1}(r)$

with the amplitude $A_{u}$, the initial frequency $\omega_{1}[\mathrm{rad} / \mathrm{s}]$, the last frequency $\omega_{2}[\mathrm{rad} / \mathrm{s}]$, the experimental time $M[\mathrm{~s}]$, the initial delay $\theta_{0}$ [rad] and the sampling time $T_{\text {sam }}$ [s]. Furthermore, due to the computational cost, the reduced models $\bar{G}_{m}(s)\left(m=1, \cdots, n_{y}\right)$ are constructed from the full-order model $G_{m}(s)$ via the balanced realization [31] keeping the controllability and observability.

In order to confirm the validity of the identified models $G_{m}(s)$ and $\bar{G}_{m}(s)$, the FIT ratio

$$
\mathrm{FIT}:=\left(1-\sqrt{\frac{\Sigma\left(\hat{y}_{m}(k)-y_{m}(k)\right)^{2}}{\Sigma\left(y_{m}(k)-E\left[y_{m}(k)\right]\right)^{2}}}\right) \times 100
$$

is evaluated for the actual output components $y_{m}(k)$ and the model output components $\hat{y}_{m}(k)$ where the symbol $E[\bullet]$ denotes expectation.

Table I summarizes the relations between the proposed visual modeling and the other types of modeling. Only the proposed visual modeling captures the whole dynamics of the liquid sloshing and is linked to design of implementable controllers in non-planar sloshing cases as well as in planar sloshing cases ${ }^{2}$. Note that some approximation techniques make us cross the modeling and the control design in Table I. For example, an equivalent mechanical modeling and an control design in input-output/linear systems \& control can be crossed via a linear approximation technique [2].

\footnotetext{
${ }^{2}$ A physical modeling along the context [32] may capture the whole dynamics and be linked to a damping injection into the tank dynamics. However, such modeling is not yet reported for liquid sloshings.
}

\section{B. Experimental setup (Modeling)}

Figure 1 shows an appearance of the experimental setup and Figure 2 shows the system configuration. The control part is mainly constructed with a real-timed PC (Linux, 2.66 [GHz], $32[\mathrm{~b}])$ with the sampling rate $1 / T_{\text {sam }}=15[\mathrm{~Hz}]$, a D/A board (12 [b]), a counter board (24 [b]) and an image capture board (RGB, $8 \times 8 \times 8[\mathrm{~b}])$. The actuation part is constructed with a DC motor (110 [W], $0.183[\mathrm{Nm} / \mathrm{A}])$, a reduction gear $(31.155[\mathrm{Nm} / \mathrm{Nm}])$ and a current servo amplifier (1.5 [A/V]). The input voltage is saturated at $\pm 5.0[\mathrm{~V}]$. The detection part is constructed with a digital camera $(640 \times 480$ [pixel] $)$ under a room light $(25[1 \mathrm{x}])$.

The plant is constructed with a tank (glass, width 450 [mm] $\times$ long $180[\mathrm{~mm}] \times$ height $300[\mathrm{~mm}]$ ), water (blue, 0.998 $\left[\mathrm{g} / \mathrm{mL}\left(20^{\circ} \mathrm{C}\right)\right], 8.10[\mathrm{~L}]$, depth $\left.120[\mathrm{~mm}]\right)$, liquid paraffin (colorless, $0.868\left[\mathrm{~g} / \mathrm{mL}\left(20{ }^{\circ} \mathrm{C}\right)\right], 12.15[\mathrm{~L}]$, depth $180[\mathrm{~mm}]$ ) and a cart which is fixed to both the tank and the camera. The driving torque of the DC motor is converted to the driving force for the tank (and the cart) dynamics via a rack and a pinion (diameter $100[\mathrm{~mm}]$ ). The static gain from the input voltage to the driving force is $171.04[\mathrm{~N} / \mathrm{V}](=0.183 \times 1.5 \times$ $31.155 / 0.05[\mathrm{~N} / \mathrm{V}])$.

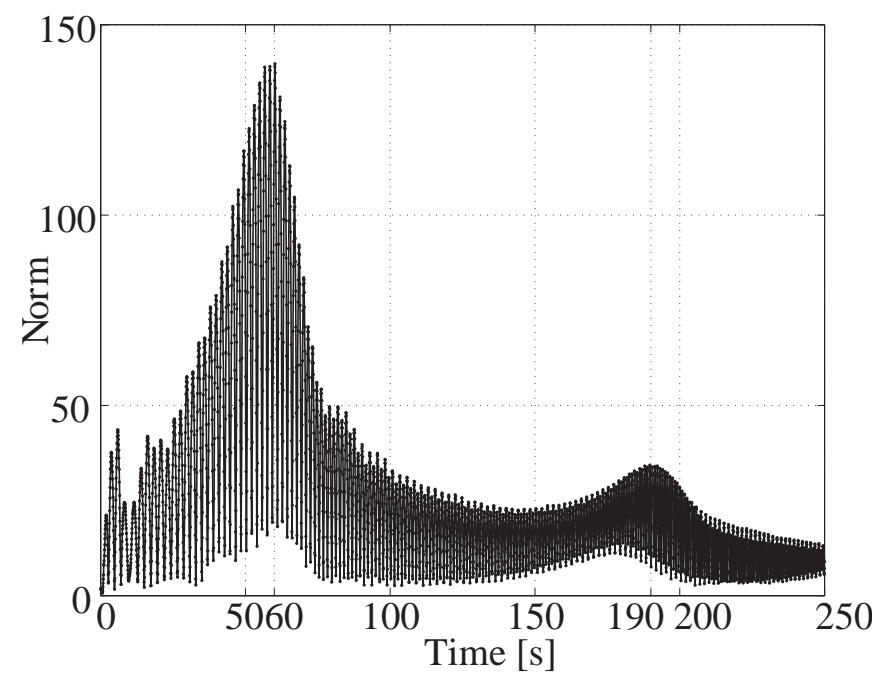

Fig. 6. Time-response of the norm.

\section{Experimental results and discussion (Modeling)}

Figure 3 shows an example image and Figure 4 shows the corresponding liquid surface $\partial \Omega$ by the input (4) whose parameters are $A_{u}=0.9[\mathrm{~V}], \omega_{1}=2 \pi 0.175[\mathrm{rad} / \mathrm{s}], \omega_{2}$ $=2 \pi 6.0[\mathrm{rad} / \mathrm{s}], M=2700(=60 \times 45)[\mathrm{s}]$ and $\theta_{0}=$ $-1 / 2 \pi$ [rad]. The thresholds are $\left(r_{1}, r_{2}, g_{1}, g_{2}, b_{1}, b_{2}\right)=$ $(12,100,12,170,150,255)$. The liquid surface $\partial \Omega$ in the example image is no longer a plane. The dots in Figure 4 depict the extracted feature and is used to calculate the output components (2). The solid line, the dashed line, and the dash-dot line depict the 9 th degree polynomial $\left[y_{1}, \cdots, y_{10}\right]^{\mathrm{T}}$, the cubic polynomial $\left[y_{1}, \cdots, y_{4}\right]^{\mathrm{T}}$, and the affine polynomial $\left[y_{1}, y_{2}\right]^{\mathrm{T}}$, respectively. Clearly, the higher degree polynomial $y$ better approximates the liquid surface $\partial \Omega$.

Figure 5 shows the time-response of the output components $y_{1}, \cdots, y_{10}$ by the same input (4). Not only the planar sloshing 

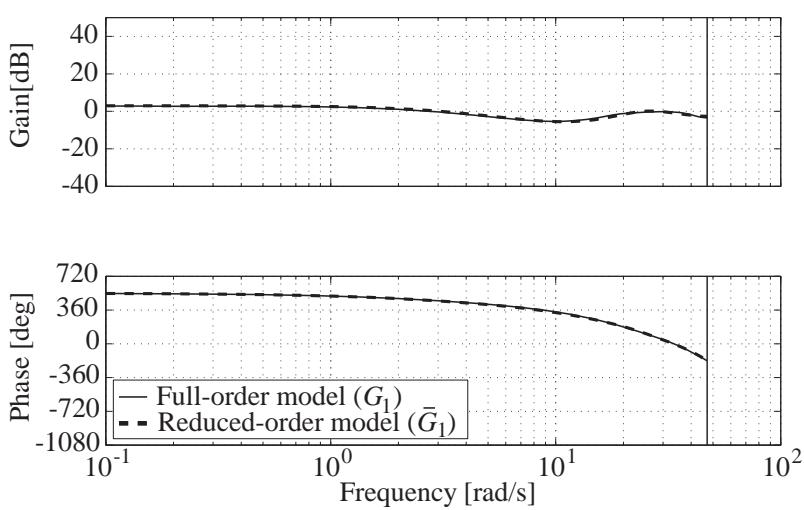

Fig. 7. Bode plots of $G_{1}(s)$ and $\bar{G}_{1}(s)$.
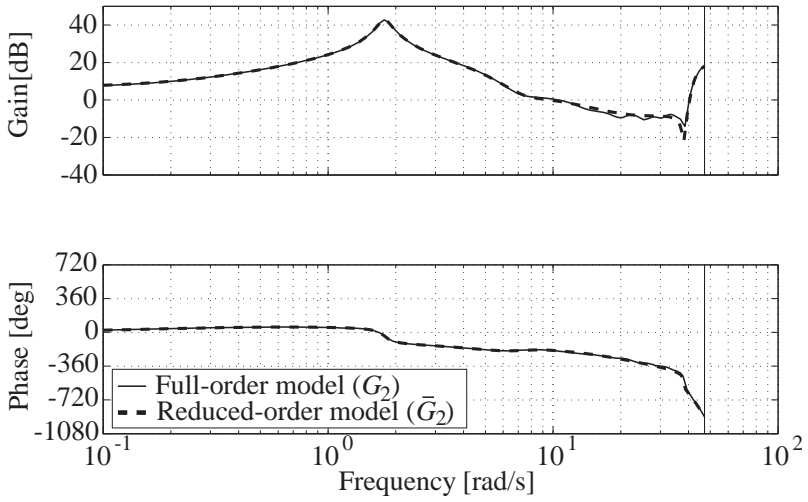

Fig. 8. Bode plots of $G_{2}(s)$ and $\bar{G}_{2}(s)$.
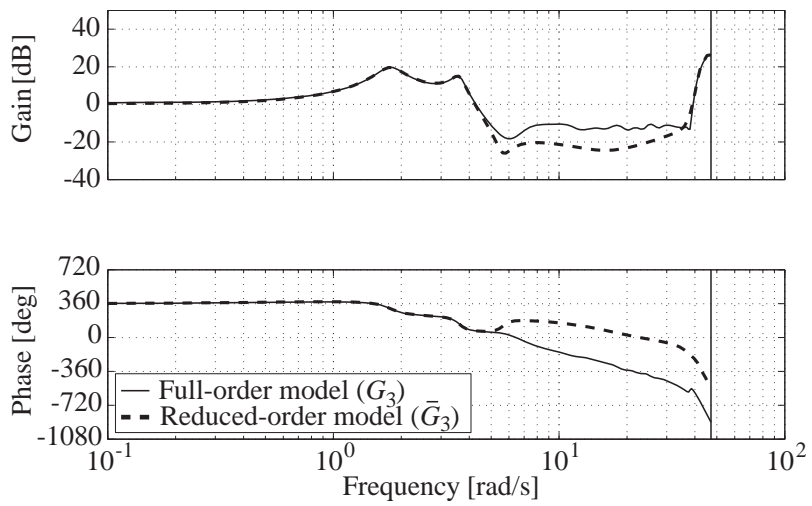

Fig. 9. Bode plots of $G_{3}(s)$ and $\bar{G}_{3}(s)$.
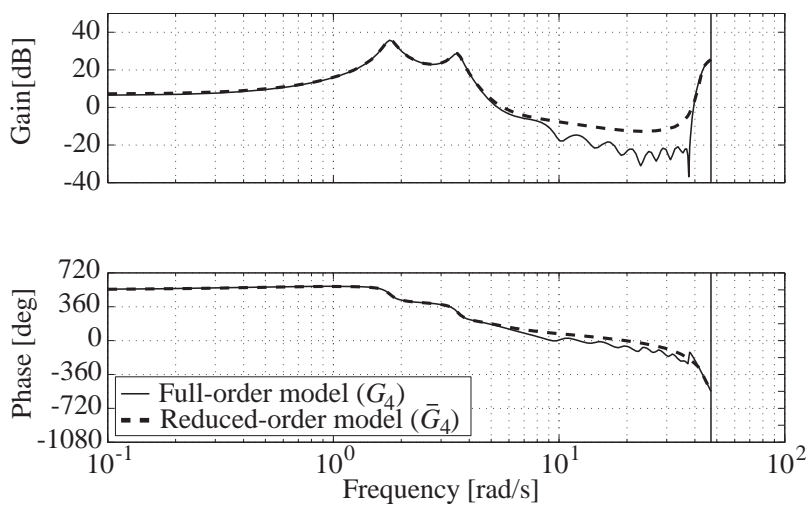

Fig. 10. Bode plots of $G_{4}(s)$ and $\bar{G}_{4}(s)$. corresponding to the components $y_{1}(k)$ and $y_{2}(k)$, but also the non-planar sloshing corresponding to the other components $y_{3}(k) \cdots y_{10}(k)$ are clearly measured on-line. The odd-degree components such as the linear polynomial's component $y_{2}$ and the cubic polynomial's component $y_{4}$ are much more dominant than the even-degree components such as the constant polynomial's component $y_{1}$ and the quadratic polynomial's component $y_{3}$. This is due to the mass conservation of the water since every even-degree polynomial is symmetric with respect to a vertical center line.
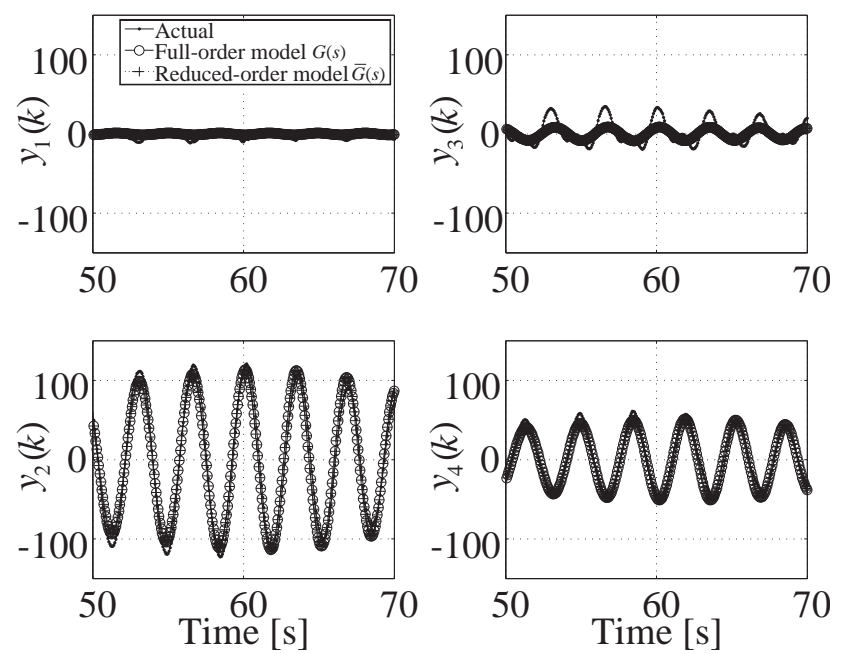

Fig. 11. Time-response of the output components $y_{m}(k)$ and $\hat{y}_{m}(k)(50-$ $70[s])$.
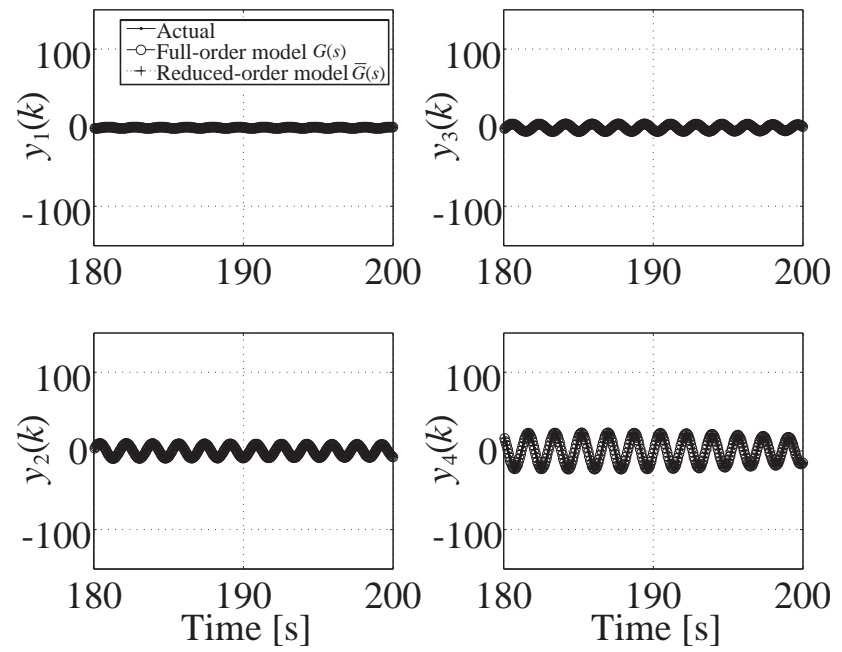

Fig. 12. Time-response of the output components $y_{m}(k)$ and $\hat{y}_{m}(k)(180-$ $200[\mathrm{~s}])$.

Figure 6 shows the time-response of the norm of the output $y(r, k)$ in case of $n_{y}=10$

$$
\|y(r, k)\|=\sqrt{y_{1}^{2}(k)+y_{2}^{2}(k)+\cdots+y_{10}^{2}(k)}
$$

by the same input (4). In Figure 5, every output component $y_{m}$ has its own peaks but the norm (5) has mainly two extremes around 60 [s] and around 190 [s] where the output components $y_{2}$ and $y_{4}$ are dominant, respectively. Thus, in the following, the output components $y_{1}, \cdots, y_{4}$ are used for control and the norm (5) is used for evaluation. 


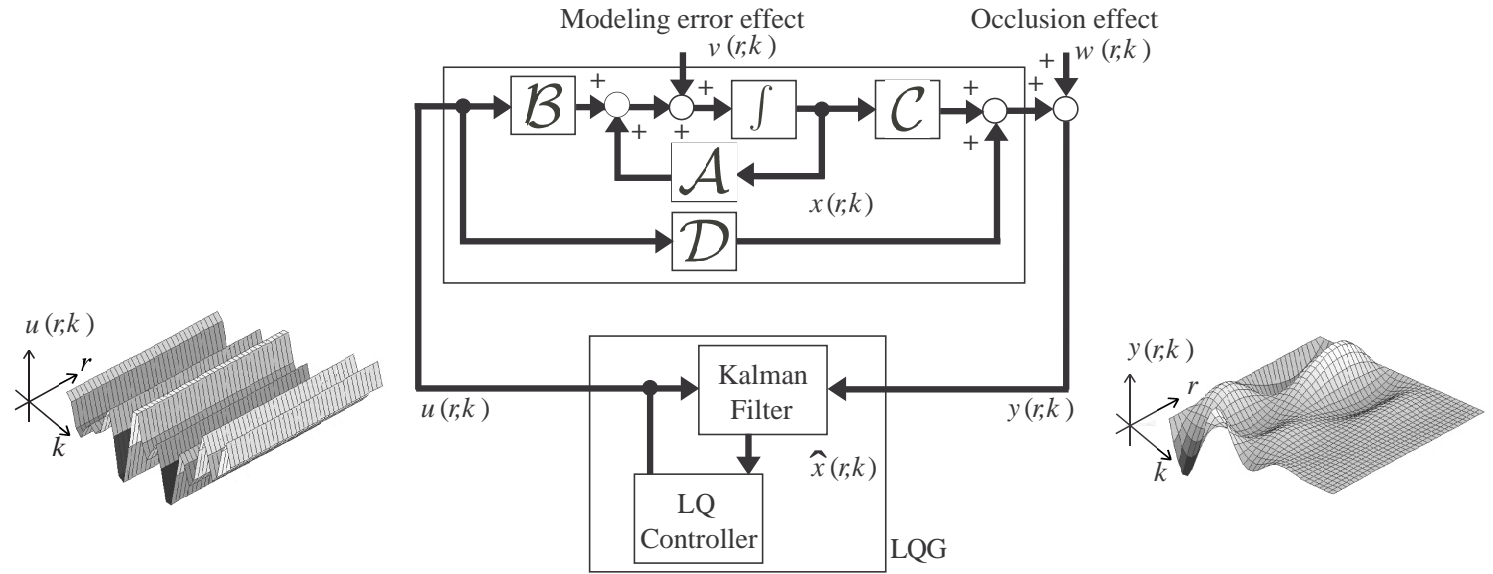

Fig. 13. Block diagram for the SIMO model with the LQG controller.

Figures 7-10 show Bode plots of the identified models $G_{m}(s)$. Every maximum gain for the even-degree components $y_{1}$ and $y_{3}$ are much smaller than that of the odd-degree components $y_{2}$ and $y_{4}$.

The identified models $G_{2}(s)$ and $G_{4}(s)$ have the peak frequencies around $0.285[\mathrm{~Hz}](1.79[\mathrm{rad} / \mathrm{s}])$ and around $0.567[\mathrm{~Hz}](3.56[\mathrm{rad} / \mathrm{s}])$, respectively. Figures 7-10 also show Bode plots of the reduced models $\bar{G}_{m}(s)$. In the lower frequency region, both models $G_{m}(s)$ and $\bar{G}_{m}(s)$ are very close to each other. In this paper, the higher frequency region is not of importance.

Figure 11 and Figure 12 show the time-response of the model output component $\hat{y}_{m}(k)$ by the same input (4). The solid line with the circles depicts the model output component $\hat{y}_{m}(k)$ of the identified model $G_{m}(s)$, the dashed line with the crosses depicts the model output component $\hat{y}_{m}(k)$ of the reduced model $\bar{G}_{m}(s)$, and the solid line with the dots depicts the actual output component $y_{m}(k)$. The modeling error effect between $\hat{y}(r, k)$ and $y(r, k)$ given by Figure 11 and Figure 12 are taken into account by the state disturbance $v(r, k)$ in the state equation (3).

Table II shows the FIT ratio between the actual output component $y_{m}(k)$ and the model output components $\hat{y}_{m}(k)$ of the identified models $G_{m}(s)$ and $\bar{G}_{m}(s)$. All FIT ratios successfully achieve positive values. Also, the FIT ratios for the odd-degree components $y_{2}(k)$ and $y_{4}(k)$ are quite high. Overall, the validity of the identified models $G_{m}(s)$ and $\bar{G}_{m}(s)$ is confirmed.

The proposed visual modeling is a new type and directly applicable to a large class of continuous systems (e.g., a vibrating nonuniform beam and a swimming fish $^{3}$ ) that are too difficult for the other types of modeling as shown in Table I. The identified model by the proposed visual modeling is quite useful as long as the input-output signal mapping can be "almost linear" between the polynomial spaces. First, although there is no physical parameter identification, the identified model will provide dynamical simulations and vibration absorber designs even if the camera images cannot be used for

\footnotetext{
${ }^{3}$ The input of the nonuniform beam can be the load and the output can be the whole vertical shape of the centerline. The input of the fish can be the whole horizontal shape of the centerline and the output can be the straight running velocity.
}

on-line control (such as in popular high-speed cameras). Note that a small-scale (or large-scale) setup based on the similitude law [8] using the physical parameters can equip the camera, the (laser sheet) lights and the millers when the full scale setup does not equip them. Second, if the camera images can be used for on-line control, the identified model also will provide controller designs as discussed in the next section.

TABLE II

FIT RATIO OF THE IDENTIFIED MODELS $G_{m}(s)$ AND $\bar{G}_{m}(s)$

\begin{tabular}{c||c|c}
\hline & Full-order & Reduced-order \\
$\bar{G}_{m}(s)[\%]$ & $(s)[\%]$ \\
\hline 1 & 14.04 & 13.81 \\
2 & 82.03 & 82.03 \\
3 & 25.94 & 25.61 \\
4 & 81.12 & 80.38 \\
\hline
\end{tabular}

\section{VISUAL FEEDBACK IN THE PRESENCE OF OCCLUSION}

In this section, the input-output modeling (system identification) on polynomial space is linked to design of implementable controllers numerically and experimentally. Furthermore, the camera occlusion effects are rejected by executing state estimation on polynomial space instead of any image processing technology.

\section{A. Experimental method (Control)}

In general, occlusion is an important issue in visual feedbacks. The existing visual feedbacks are helped by image processing technology which needs to assume the range of the shape and size of the visual obstacles. However, in the framework of visual systems \& control on polynomial space, the camera occlusion effects can be modeled as output disturbance to be rejected by control systems technology.

In this paper, as a proposed control approach, a Linear Quadratic and Gaussian (LQG) controller is designed for the reduced single-input multiple-output (SIMO) model $\bar{G}(s):=$ $\left(\bar{G}_{1}(s), \bar{G}_{2}(s), \bar{G}_{3}(s), \bar{G}_{4}(s)\right)^{\mathrm{T}}$ on polynomial space. Also, as a conventional control approach, another LQG controller is designed for the SISO model $\bar{G}_{2}(s)$. The conventional controllers in planar sloshing cases [5] correspond to the LQG controller for the SISO model $\bar{G}_{2}(s)$ because the planar 


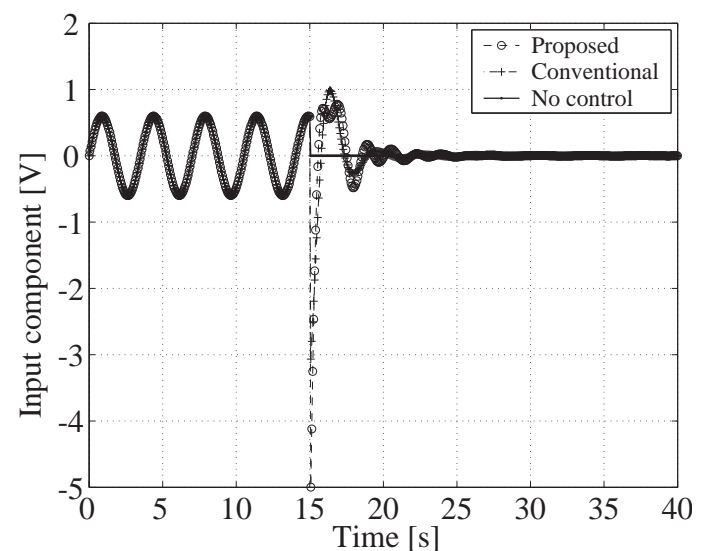

Fig. 14. Time-response of the input component (Condition L).

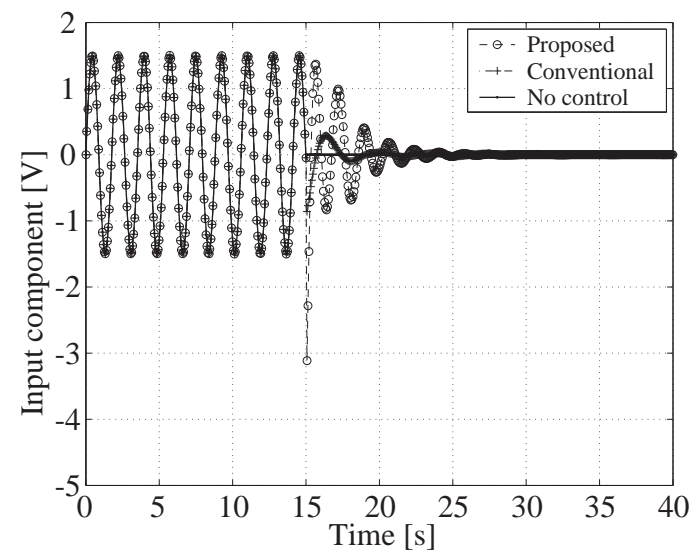

Fig. 15. Time-response of the input component (Condition $\mathrm{H}$ ).

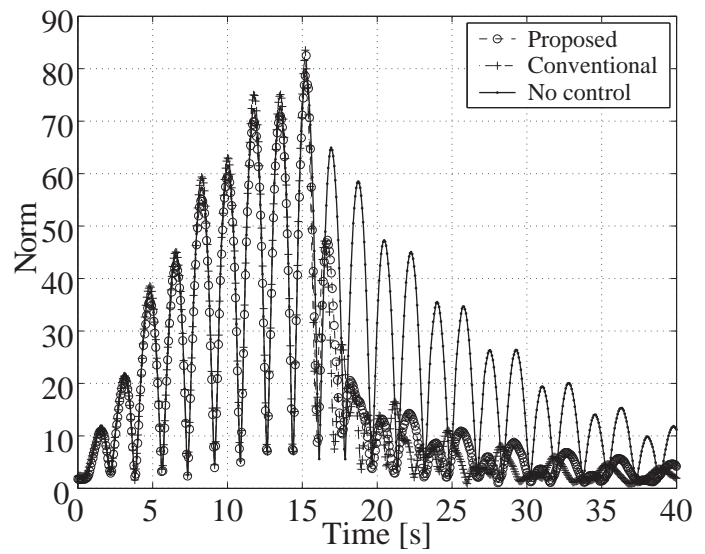

Fig. 16. Time-response of the norm (Condition L).

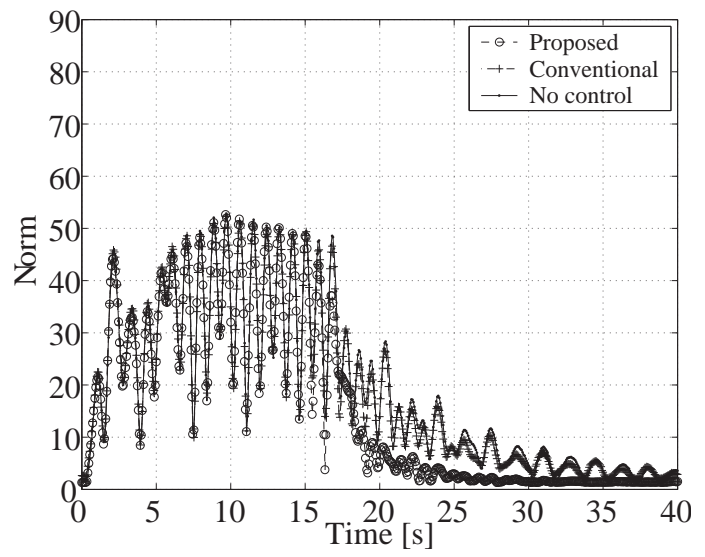

Fig. 17. Time-response of the norm (Condition H). sloshing is expressed as $\left[y_{1}, y_{2}\right]^{\mathrm{T}}$ but the component $y_{1}$ is very small and negligible due to the mass conservation of the water as discussed in Section III.

First, in the absence of occlusion, the control performance of these approaches are experimentally compared with each other and also with that of no control approach. Second, the control performance is investigated again in the presence of occlusion which is a student's left hand and its effects are modeled as output disturbance $w(r, k)$ expressed as $\left[w_{1}(k), w_{2}(k), w_{3}(k), w_{4}(k)\right]^{\mathrm{T}}$ for the SIMO model $\bar{G}(s)$ and $w(r, k)$ expressed as $\left[w_{1}(k)\right]$ for the SISO model $\bar{G}_{2}(s)$, respectively.

Before the controls begin, the initial state is made by the following sinusoidal input for a fixed time (15.00 [s]) starting from a steady horizontal surface:

Condition L: A planar sloshing with a dominant $y_{2}(k)$

$$
u(r, k)=0.55 \sin \left(2 \pi 0.285 T_{\text {sam }} k\right) e_{1}(r)
$$

Condition H: A non-planar sloshing with a dominant $y_{4}(k)$

$$
u(r, k)=1.50 \sin \left(2 \pi 0.567 T_{\mathrm{sam}} k\right) e_{1}(r)
$$

These input frequencies and amplitudes are prepared based on Bode plots shown in Figures 7-10.

Just after the above sinusoidal inputs end, the control in each approach begins. The weights of the LQG design [26]

$$
\begin{aligned}
\Sigma_{k=0}^{\infty}\left(\langle x(r, k), x(r, k)\rangle_{Q_{f}}+\langle u(r, k), u(r, k)\rangle_{R_{f}}\right), & \\
E & {\left[\left[\begin{array}{c}
v_{1}(k) \\
\vdots \\
v_{n_{x}}(k)
\end{array}\right]\left[\begin{array}{c}
v_{1}(l) \\
\vdots \\
v_{n_{x}}(l)
\end{array}\right]\right]=R_{e} \delta(k-l), } \\
E & {\left[\left[\begin{array}{c}
w_{1}(k) \\
\vdots \\
w_{n_{y}}(k)
\end{array}\right]\left[\begin{array}{c}
w_{1}(l) \\
\vdots \\
w_{n_{y}}(l)
\end{array}\right]\right]=Q_{e} \delta(k-l) }
\end{aligned}
$$

are $Q_{f}=0.0001 \times I_{31}, R_{f}=1000, Q_{e}=50000 \times I_{31}, R_{e}=$ ones(31) for the SIMO model $\bar{G}(s)$ in Figure 13 and $Q_{f}=$ $0.00002 \times I_{8}, R_{f}=500, Q_{e}=50000 \times I_{8}, R_{e}=\operatorname{ones}(8)$ for the SISO model $\bar{G}_{2}(s)$ where, via the balanced realization [31] keeping the controllability and observability, the SIMO model $\bar{G}(s)$ is the 31-dimensional system and the SISO model $\bar{G}_{2}(s)$ is the 8-dimensional system. Note that the SIMO model in Figure 13 is equivalent to the system of the state space expression (3). Here the symbol $\langle\bullet, \bullet\rangle_{X}$ denotes the weighted inner product by the matrix $X$ and the symbol ones $(n)$ denotes the $n \times n$ matrix whose components are all 1 .

\section{B. Experimental results and discussion (Control)}

Figure 14 shows the time-response of the input component in Condition L and Figure 15 shows that of the input component in Condition H. The solid line with the circles depicts the input for the SIMO model $\bar{G}(s)$ and the solid line with the crosses depicts the input for the SISO model $\bar{G}_{2}(s)$. No input saturations are seen since the maximum magnitude of the input component is -4.99 [V] just after 15.00 [s] for the SIMO model $\bar{G}(s)$ in Condition L but is still larger than -5.0 [V]. 


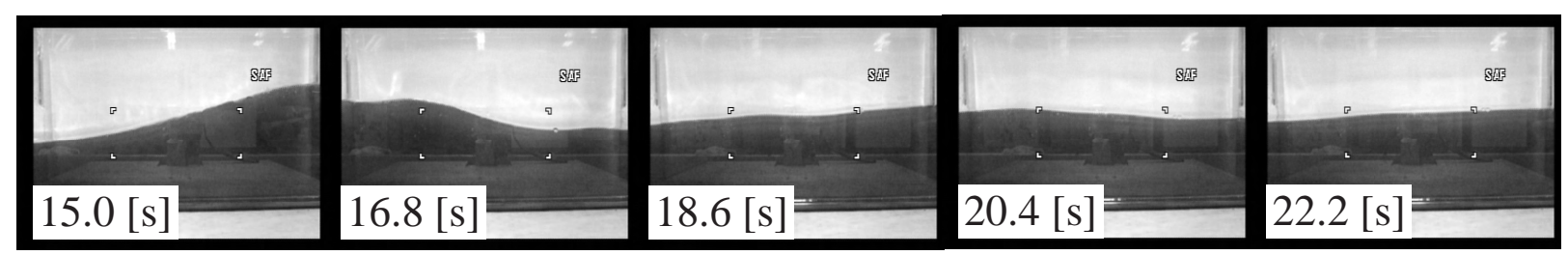

Fig. 18. Images (Condition L, Proposed).

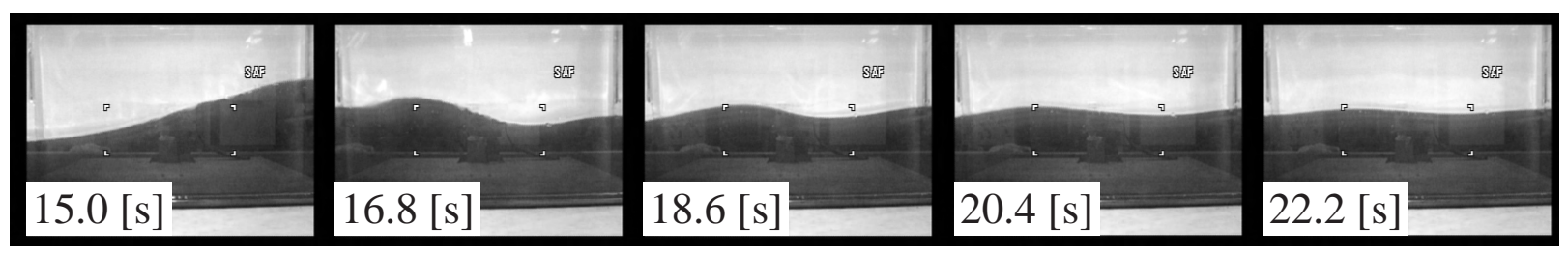

Fig. 19. Images (Condition L, Conventional).

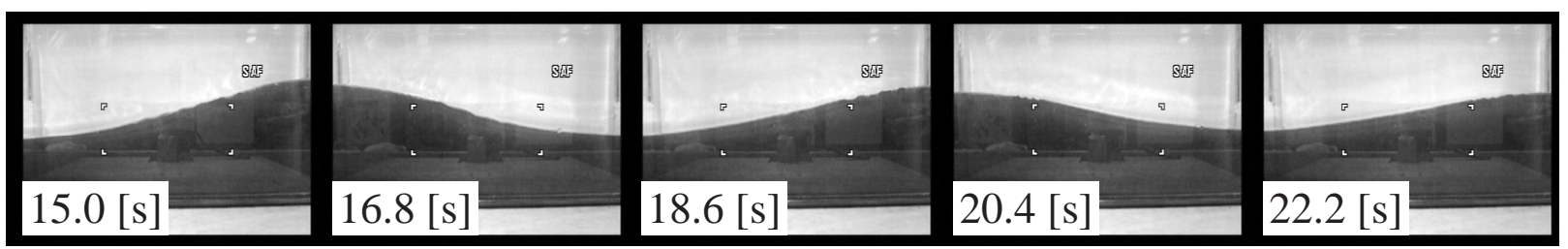

Fig. 20. Images (Condition L, No control).

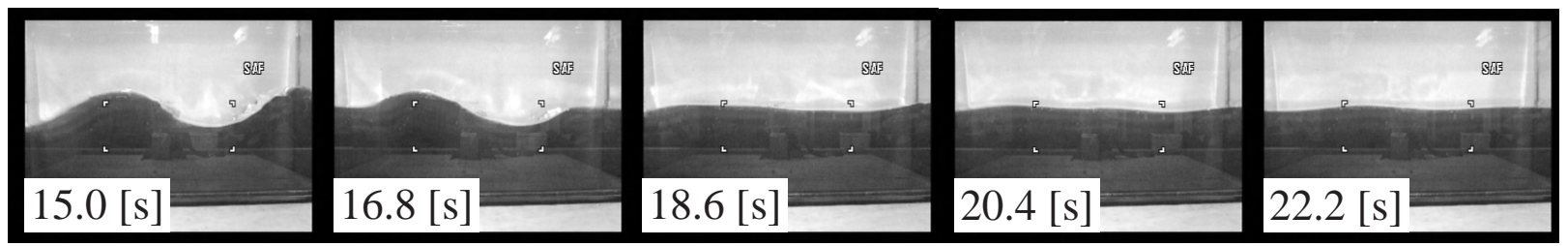

Fig. 21. Images (Condition H, Proposed).

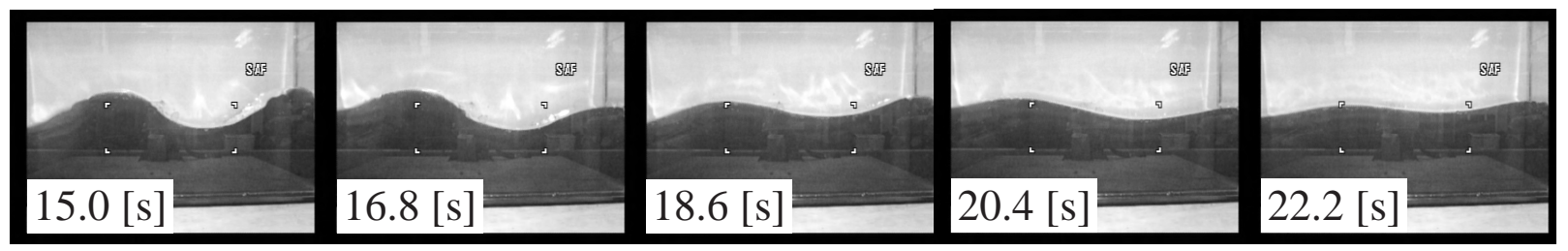

Fig. 22. Images (Condition $\mathrm{H}$, Conventional).

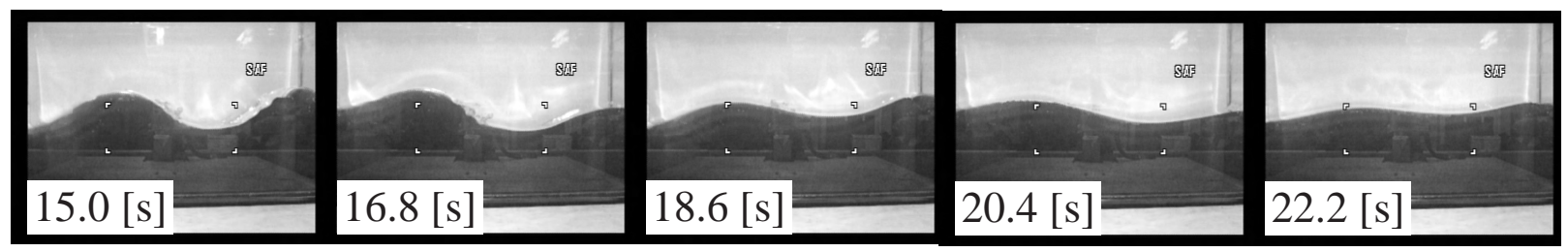

Fig. 23. Images (Condition H, No control).

Figure 16 shows the time-response of the norm (5) in Condition L and Figure 17 shows that of the norm (5) in Condition $\mathrm{H}$. To compare the control performance, a settling time $t_{s}$ is introduced as the last time when the norm is less than $15 \%$ of the initial norm at 15.00 [s] when the controls begin.

The settling times in Figure 16 are $t_{s}=7.50[\mathrm{~s}]$ by the proposed control approach, $t_{s}=6.44$ [s] by the conventional control approach and $t_{s}=21.68$ [s] by no control approach in Condition L. The proposed control approach improved the settling time by 65 [\%] compared with that by no control approach while the conventional control approach improved the settling time by 70 [\%]. In Condition L, the effectiveness of both the control approaches is confirmed.

In addition, the settling times in Figure 17 are $t_{s}=4.62$ [s] by the proposed control approach, $t_{s}=12.57$ [s] by the conventional control approach and $t_{s}=12.79$ [s] by no control approach in Condition $\mathrm{H}$. The proposed control approach improved the settling time by 63 [\%] again compared with that by no control approach. However, the conventional control approach improved the settling time just by only 2.0 [\%]. In Condition $\mathrm{H}$, the effectiveness of the proposed control approach is confirmed, but the conventional control approach fails. 


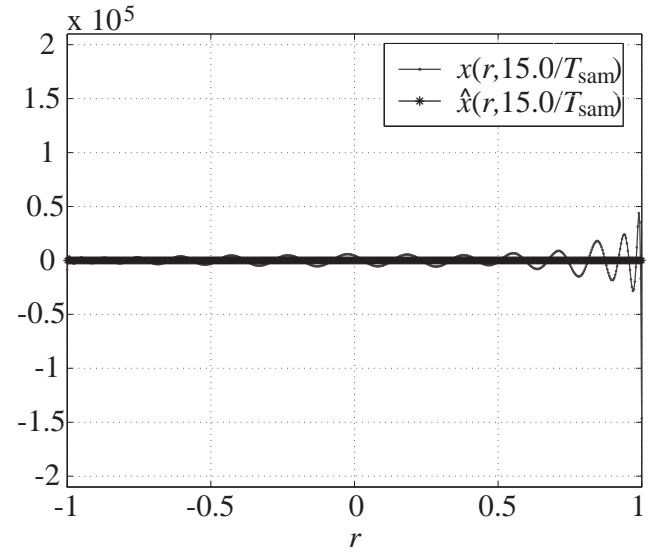

Fig. 24. The state $x\left(r, 15.0 / T_{\mathrm{sam}}\right)$ and the estimated state $\hat{x}\left(r, 15.0 / T_{\mathrm{sam}}\right)$.

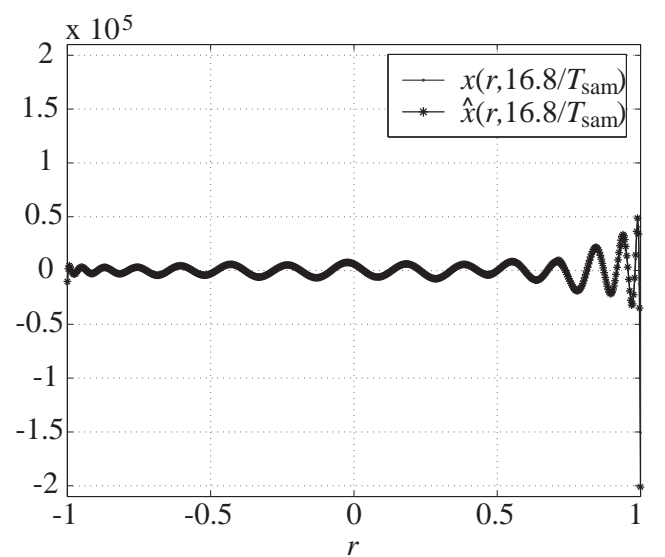

Fig. 25. The state $x\left(r, 16.8 / T_{\text {sam }}\right)$ and the estimated state $\hat{x}\left(r, 16.8 / T_{\text {sam }}\right)$.

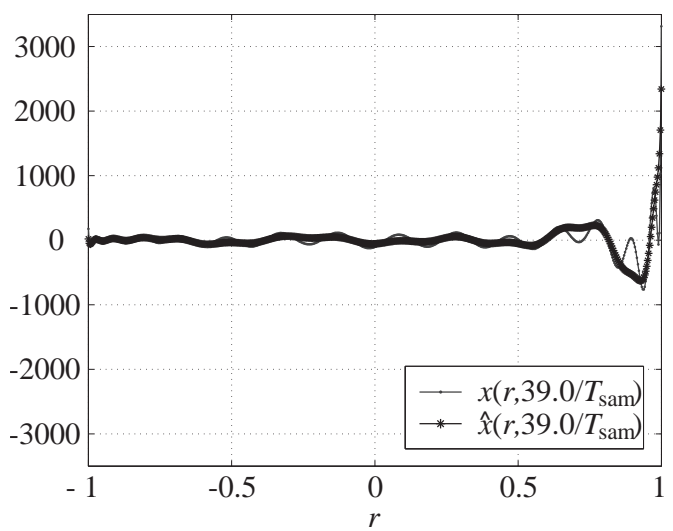

Fig. 26. The state $x\left(r, 39.0 / T_{\text {sam }}\right)$ and the estimated state $\hat{x}\left(r, 39.0 / T_{\text {sam }}\right)$.

Figures 18-23 show the example images in Condition $\mathrm{L}$ and Condition $\mathrm{H}$, respectively. Indeed, the planar sloshing is generated in Condition L while the non-planar sloshing is generated in Condition H. It is confirmed that the control performance of the proposed control approach is good even in Condition $\mathrm{H}$ where the conventional control approach is useless. Indeed, there is few difference between the images by the conventional control approach and those by no control approach in Condition $\mathrm{H}$.

In a word, the conventional control approach for the planar sloshing case does not work for the non-planar sloshing case at all. Clearly, this is because the SISO model $\bar{G}_{2}(s)$ has no peaks around the input frequency $0.567[\mathrm{~Hz}]$ in Condition $\mathrm{H}$.
In all, the effectiveness of the proposed control approach is confirmed in the absence of occlusion.

Figures 24-26 draw the state $x(r, k)$ in the SIMO model $\bar{G}(s)$ and the estimated state $\hat{x}(r, k)$ in the corresponding LQG controller at the discrete time instant $k=$ $15.0 / T_{\text {sam }}, 16.8 / T_{\text {sam }}, 39.0 / T_{\text {sam }}$, respectively. These figures verify the LQG design for all of the experiments in this paper. The state $x(r, k)$ is not visualizable in general and thus every state in these figures is made by just a numerical simulation with the same experimental conditions as in Condition $\mathrm{H}$. The polynomial space makes it possible to draw this 31dimensional state directly though coordinate spaces can not allow us to draw even four-dimensional state.

Although the initial state $x\left(r, 15 / T_{\text {sam }}\right)$ differs from the initial estimated state $\hat{x}\left(r, 15 / T_{\text {sam }}\right)$ which is defined as a zero constant polynomial in Figure 24, the two states become closer to each other in both Figure 25 and Figure 26. It should be noted that the state $x\left(r, 16.8 / T_{\text {sam }}\right)$ is still approximated well by the estimated state $\hat{x}\left(r, 16.8 / T_{\text {sam }}\right)$ even when the norm (5) is at the transient state as shown in Figure 17. These results imply that a state estimation on polynomial space is achieved well by the Kalman filter which is different from all the other Kalman filters [33] [34] on coordinate space in the conventional visual feedbacks. Also, at the almost steady state in Figure 26, both states converge to the zero constant polynomial. That is, the origin in the polynomial space is asymptotically stabilized. The LQG design used in the experiments are justified numerically.

In the presence of occlusion, Figure 27 and Figure 28 show the time-response of the input component in Condition $\mathrm{L}$ and Condition H, respectively. Figure 29 and Figure 30 show the time-response of the norm (5) in Condition L and Condition $\mathrm{H}$, respectively. In comparison with Figure 16 and Figure 17, the measured outputs $y$ are strongly effected by the occlusion which is the student's hand as shown in Figure 31 and Figure 32. Clearly, such camera occlusion effects can be modeled as the output disturbance $w(r, k)$ which may lose the control performance at least.

However, in both Condition $\mathrm{L}$ and Condition $\mathrm{H}$, the camera occlusion effects are successfully rejected by the same LQG controller without any modification. That is, due to the Kalman filter on polynomial space, the control performance with the camera occlusion effects are the almost same as that without occlusion. Note that no image processing technology was added to solve the occlusion problem. In all, in the presence of occlusion, the effectiveness of the proposed control approach is confirmed. The ARX method and the LQG design in this paper are just examples and various existing modeling and control methods on coordinate space are directly applicable on polynomial space.

\section{CONCLUSION}

This paper proposes a novel approach for camera based modeling and control and the validity is confirmed by liquid sloshing experiments. In existing types of modeling, nonplanar sloshings are not modeled well for control because all signal mappings from the input (the driving force for the tank) 


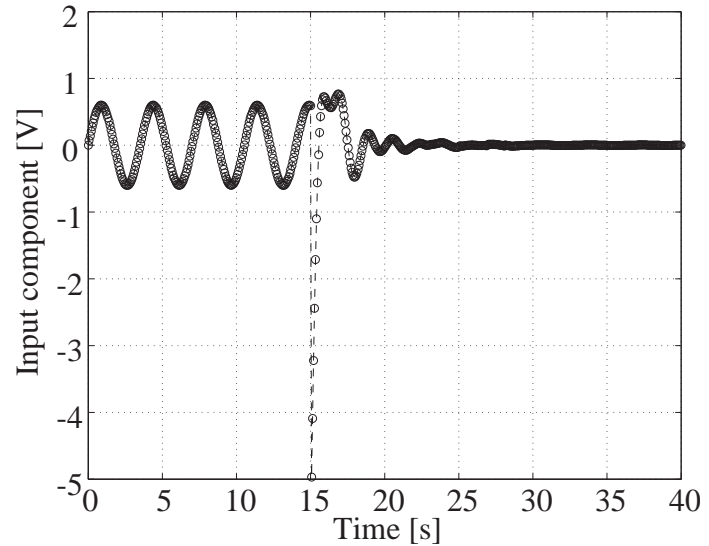

Fig. 27. Time-response of the input component (Condition L, Proposed + Occlusion).

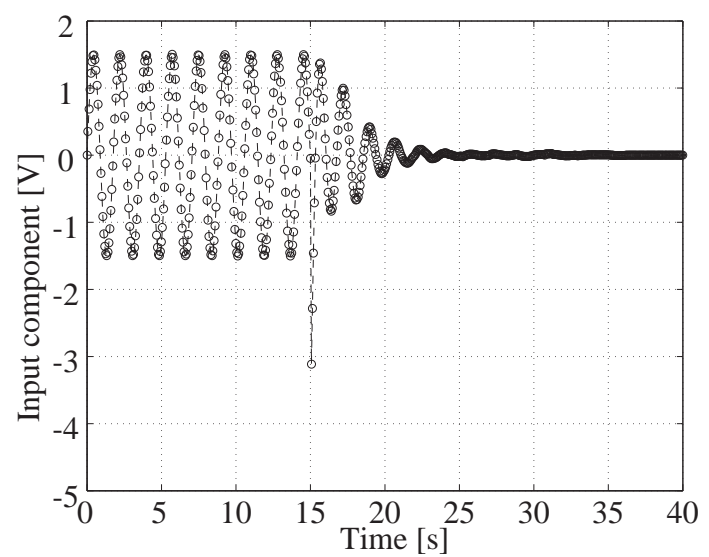

Fig. 28. Time-response of the input component (Condition H, Proposed + Occlusion).

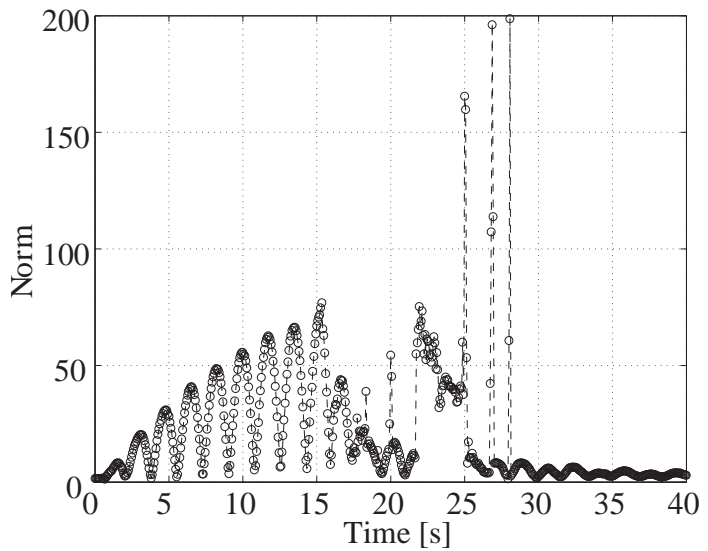

Fig. 29. Time-response of the norm (Condition L, Proposed + Occlusion).

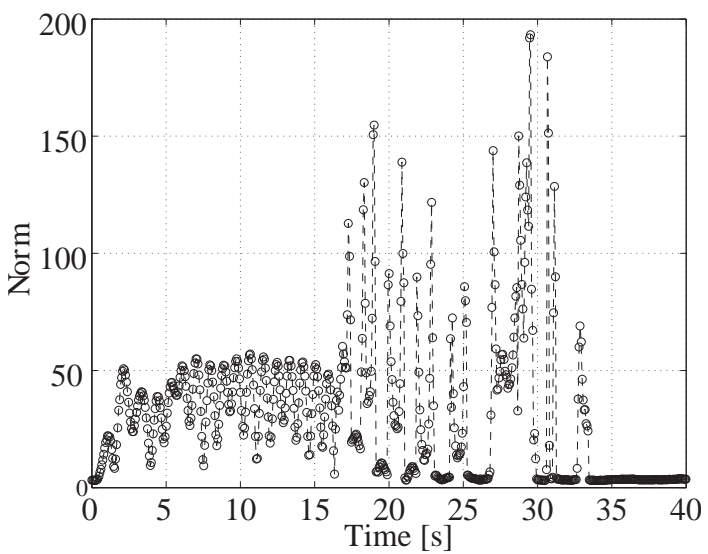

Fig. 30. Time-response of the norm (Condition H, Proposed + Occlusion). to any liquid surface displacement are strongly nonlinear on coordinate space. However, in the proposed visual modeling, since the whole shape of the liquid surface is the object in a polynomial space as well as the input and the state, a signal mapping from the same input to the whole shape of the liquid surface can be "almost linear", that is, the nonplanar sloshings are modeled well for control. The proposed approach will solve many modeling and control problems of a large class of continuous systems that are too difficult for existing approaches using coordinate spaces.

More precisely, first, not only the whole shape of the liquid surface corresponding to the output, but also the input and the state are introduced as time-varying polynomials. The output is a visible polynomial measured by a camera and the input is a visualizable polynomial. The state is never visualizable but exists and causes the liquid sloshing.

Second, the proposed visual modeling captures both the planar and the non-planar sloshing cases. As long as the signal mapping between the polynomial spaces are "almost linear", the mapping is identified as an LTI model which is quite useful because the identified LTI model will provide dynamical simulations and vibration absorber designs without physical parameter identification even if camera images cannot be used for on-line control.

Furthermore, even in the presence of occlusion, the proposed visual modeling is linked to implementable controllers. The actual liquid surface is controlled well by executing a control design and a state estimation on polynomial space without adding any image processing technology.

Although chaotic sloshing cases with overturned waves are not yet captured in this paper, so many existing modeling and control methods (e.g., ARX, N4SID, LQG, $H_{\infty}$ and so on) constructed on coordinate space are now applicable to so many continuous systems from the viewpoint of the polynomial space.

Following the proposed visual modeling in this paper, one may propose to change the space. Indeed, instead of the polynomial space, another Hilbert space, whose basis is from the Bessel functions in physical modeling, may lead to a faster control computation in the liquid sloshing problems. However, such a basis is specific to each continuous system and also needs the exact physical parameter identification which actually boils down to physical modeling. In contrast, the proposed visual modeling on polynomial space is directly applicable to a large class of continuous systems as long as the input-output signal mapping between the polynomial spaces can be "almost linear". Also, there is no need of physical parameter identification. In this sense, the proposed visual modeling is robust in the presence of parameter perturbation and uncertainty such as in a vibrating nonuniform beam and a swimming fish.

This paper is motivated by application. There are no theorems. Nevertheless, this paper extends the range of application of various existing modeling and control methods by a choice of the new input and output spaces (domain and co-domain) without thinking any new type of controllers (mappings). The authors believe that this paper successfully highlights an original role of control systems technology. 


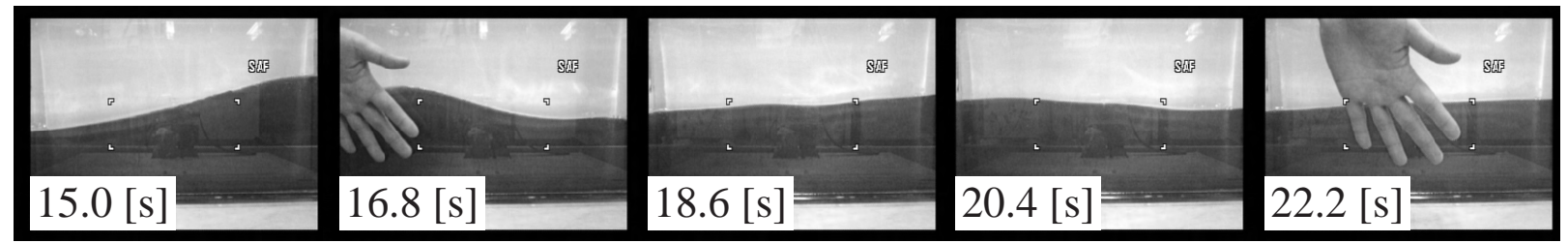

Fig. 31. Images (Condition L, Proposed + Occlusion).

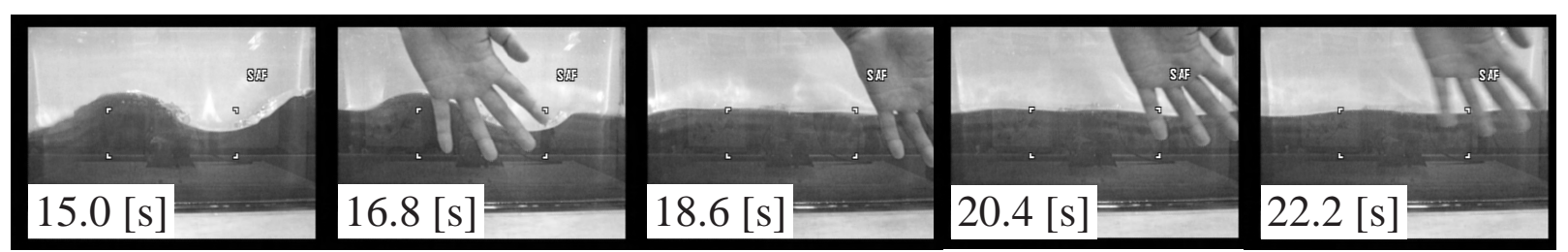

Fig. 32. Images (Condition H, Proposed + Occlusion).

\section{ACKNOWLEDGMENT}

We would like to express our gratitude to Mr. Kazuyuki Nagai, Prof. Hiroyuki Suga and Prof. Yuichi Chida.

\section{REFERENCES}

[1] Mohammad Ali Goudarzi. Autoparametric resonances of elastic structures coupled with two sloshing modes in a square liquid tank. ASME Journal of Computational and Nonlinear Dynamics, 8(1):381-390, 2013.

[2] Bogdan Robu, Lucie Baudouin, Christophe Prieur, and Denis Arzelier. Simultaneous Hinf vibration control of fluid/plate system via reduced order controller. IEEE Transactions on Control Systems Technology, 20(3):700-711, 2012.

[3] S. Sakai, K. Osuka, T. Maekawa, and M. Umeda. Robust control systems of a heavy material handling agricultural robots. IEEE Transactions on Control Systems Technology, 15(6):1038-1048, 2007.

[4] Oleg Yu. Emanouilov (Imanuvilov). Controllability of evolution equations of fluid dynamics. In International Congress on Mathematitians, pages 13211338, 2006

[5] R. A. Ibrahim. Liquid Sloshing Dynamics: Theory and Applications. Cambridge University Press, 2005.

[6] O. M. Faltinsen and A. N. Timokha. Sloshing. Cambridge University Press, 2009.

[7] O. M. Faltinsen and Alexander N. Timokha. On sloshing modes in a circular tank. Journal of Fluid Mechanics, 695:467-477, 2012.

[8] V. I. Arnold. Mathematical Methods of Classical Mechanics. SpringerVerlag, New York, second edition, 1989.

[9] H. K. Khalil. Nonlinear Systems. Macmillan Publishing Company, New York, third edition, 1996.

[10] M. Krsti, I. Kanellakopoulos, and P. Kokotovi. Nonlinear and Adaptive Control Design. John Wiley Sons, 1995.

[11] K. Yano and K. Terashima. Sloshing suppression control of liquid transfer systems considering a 3-D transfer path. IEEE/ASME Transactions on Mechatoronics, 10(1):8-16, 2005.

[12] Milovan Peric, Tobias Zorn, Ould el Moctar, Thomas E. Schellin, and Yong-Soo Kim. Simulation of sloshing LNG tanks. ASME Journal of Offshore Mechacnics and Arctirce Engineering, 131(3):031101-11, 2009.

[13] E. R. Williams, D. Haddad, G. Geneves, P. Gournay, C. Hauck, F. Villar, R. L. Steiner, and R. Liu. Balance pan damping using rings of tuned sloshing liquids. IEEE Transactions on Instrumentation and Measurement, 58:949-954, 2009

[14] Shailaja Kurode, Sarah K. Spurgeon, Bijnan Bandyopadhyay, and P. S.
Gandhi. Sliding mode control for slosh-free motion using a nonlinear sliding surface. IEEE/ASME Transactions on Mechatoronics, 18(2):714- 724, 2013. [15] Hanz Richter. Motion control of a container with slosh: Constrained sliding mode approach. ASME Journal of Dynamic Systems, Measurement and Control, 132(3):031002-10, 2010.

[16] B. Bandyopadhyay, P. S. Gandhi, and Shailaja Kurode. Sliding mode observer based sliding mode controller for slosh-free motion through PID scheme. IEEE Transactions on Industrial Electronics, 56(9):3432- 3441, 2009. [17] K. Yano and K. Terashima. Robust liquid container transfer control for complete sloshing suppression. IEEE Transactions on Control Systems Technology, 9(3):483-493, 2001.

[18] John T. Feddema, Clark R. Dohrmann, Gordon G. Parker, Rush D. Robinett, Vicente J. Romero, and Dan J. Schmitt. Control for sloshfree motion of an open container. In Proc. of IEEE, pages 29-36, 1997.

[19] Brice Pridgen, Kun Bai, and William Singhose. Slosh suppression by robust input shaping. In Proc. of IEEE Conference on Decision and Control, pages 15-17, 2010.

[20] M. Utsumi. A mechanical model for low-gravity sloshing in an axisymmetric tank. ASME Journal of Applied Mechanics, 71(5):724- 730, 2004.

[21] S. Arimoto. Control Theory of Non-linear Mechanical Systems: Passivitybased and Circuit-theoretic Approach. Clarendon Press, Oxford, 1996.

[22] A. J. van der Schaft. $L_{2}$-Gain and Passivity Techniques in Nonlinear Control. Springer-Verlag, London, 2000.

[23] Mattias Grundelius and Bo Bernhardsson. Control of liquid slosh in an industrial packaging machine. In International Conference on Control Applications, pages 1654-1659, 1999.

[24] P. van Overschee and B. De Moor. Subspace identification for Linear systems. Kluwer Academic Publication, 1996.

[25] L. Ljung. System Identification: Theory for the User. Prentice-Hall, Upper Saddle River, New Jersey, second edition, 1999.

[26] David Luenverger. Optimization by vector space method. John Wiley Sons, 1960 .

[27] Joseoh M. Crowley. Control of an amplifying wave on an infinite continuum. IEEE Transactions on Automatic Control, 14(5):536-539, 1969.

[28] X. W. Tangpong and Om P. Agrawal. Fractional optimal control of continuum systems. ASME Journal of Vibration and Acoustics, 131(2): 10.1115/1.3025833, 2009.

[29] B. F. Feeny and A. K. Feeny. Complex modal analysis of the swimming motion of a whiting. ASME Journal of Vibration and Acoustics, 135(2):10.1115/1.4023056, 2013.

[30] Gilbert Strang. Computational Science and Engineering. 2007.

[31] K. Zhou, J. C. Doyle, and K. Glover. Robust and Optimal Control. 
Prentice-Hall, Inc., Upper Saddle River, N.J., 1996.

[32] A. J. van der Schaft and B. M. J. Maschke. Hamiltonian formulation of distributed-parameter systems with boundary energy flow. Journal of Geometry and Physics, 42:166-194, 2002.

[33] Xiaojing Song, L.D. Seneviratne, and K. Althoefer. A Kalman filter integrated optical flow method for velocity sensing of mobile robots. IEEE/ASME Transactions on Mechatronics, 16(3):551 -563, 2011.

[34] S. Y. Chen. Kalman filter for robot vision: A survey. IEEE Transactions on Industrial Electronics, 59(11):4409-4420, 2012.

\begin{tabular}{|c|} 
\\
\\
PLACE \\
PHOTO \\
HERE \\
\end{tabular}

Satoru Sakai Satoru Sakai received the B.E. degree in Engineering and the M.E. and Ph.D. degrees in Agricultural Engineering from Kyoto University, Japan, in 1998, 2000 and 2003, respectively. In 2005, he joined Chiba University. In 2010, he joined Shinshu University, Japan, where he is currently an associate professor with the Department of Mechanical Engineering. From 2003 to 2005, he was a JSPS postdoctoral research fellow with the Department of Informatics, Kyoto University, and from 2004 to 2005 he was a visiting researcher with the Faculty of Electrical Engineering, Twente University, The Netherlands. His research interests include robotics and physical systems \& control. He was a recipient of the Young Author Prize at IFAC 2005.

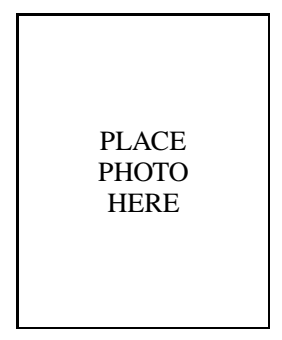

Masakazu Sato Sato Masakazu received his B.S. degrees in engineering from Shinshu University, Nagano, Japan, in 2011 and his M.S. degrees in engineering from Shinshu University, Nagano, Japan, in 2013. 